\title{
Finite Temperature Static Charge Screening in Quantum plasmas
}

\author{
B. Eliasson ${ }^{1}$ and M. Akbari-Moghanjoughi ${ }^{2}$ \\ ${ }^{1}$ SUPA, Physics Department, John Anderson Building, \\ University of Strathclyde, Glasgow G4 ONG, Scotland, UK \\ ${ }^{2}$ Faculty of Sciences, Department of Physics, \\ Azarbaijan Shahid Madani University, 51745-406 Tabriz, Iran
}

(Dated: 19 May 2015)

\begin{abstract}
The shielding potential around a test charge is calculated, using the linearized quantum hydrodynamic formulation with the statistical pressure and Bohm potential derived from finite temperature kinetic theory, and the temperature effects on the force between ions is assessed. The derived screening potential covers the full range of electron degeneracy in the equation of state of the plasma electrons. An attractive force between shielded ions in an arbitrary degenerate plasma exists below a critical temperature and density. The effect of the temperature on the screening potential profile qualitatively describes the ion-ion bound interaction strength and length variations. This may be used to investigate physical properties of plasmas and in molecular-dynamics simulations of fermion plasma. It is further shown that the Bohm potential including the kinetic corrections has a profound effect on the Thomson scattering cross section in quantum plasmas with arbitrary degeneracy.

PACS numbers: 52.30.-q,71.10.Ca, 05.30.-d
\end{abstract}

Keywords: Quantum plasma, screening, Fermi-Dirac distribution 


\section{INTRODUCTION}

Plasma-based techniques have become an important aspect of the fabrication of miniature electronic devices $[1,2]$ and of nanotechnology [3]. Theoretical models have to account for various atomic-scale interactions between charge species in dense and hot to ultra-cold plasmas. These interaction are usually beyond the simple long-range Coulomb interactions or the standard Deby-Hückel charge screening due to the complex nature of electromagnetic interactions in high-energy-density (HED) plasmas [4]. In the treatment of dense plasmas, the Thomas-Fermi approximation is sometimes not sufficient for accurate descriptions of collective phenomena, and in order to describe the essential physics of strongly interacting plasmas, one needs to go beyond the standard approximations. The study of collective interactions in quantum plasmas has a long tradition [5-12] since metals, semiconductors [13], cores of large planets [14] and dense astrophysical objects $[15,16]$ fall into this category. Obviously, the ultimate choice for plasma simulations and research are the appropriate kinetic models [17] for plasma coupled with Poisson's equation or Maxwell's equations. However, a full implementation of kinetic approach including interactions and collision terms is limited due to their complexity. There are approximate hydrodynamic models which can incorporate a variety of collective aspects such as electron quantum diffraction [18-22], finite temperature [23], electron exchange-correlation [24, 25], quantum statistical pressure [26] and spin-magnetization $[27,28]$ with much less complexity compared to the fully kinetic counterparts. Application of quantum hydrodynamic models have proven to unveil a large number of interesting physical aspects of dense plasmas $[29,30]$ which has been previously thought would only be possible using more advanced theories.

One of the interesting results of linearized quantum hydrodynamic (LQHD) theory is an attractive screening charge-potential first formulated for the screening and wake potentials in a dilute plasma where the statistical pressure was neglected [31], and was later complemented by including the statistical pressure and charge-exchange effects in the zero temperature limit [32]. The reason for the existence of an attractive potential among ions of similar charge is the electron diffraction effect resulting in the Bohm potential in the hydrodynamic model, most prominently in fluid-like descriptions of the single-electron dynamics [7, 33, 34]. However, the existence of an attractive screening force in degenerate systems has been a subject of intense debate due to conflicting results with density functional theory (DFT) [35-39]. It 
later became apparent that the inclusion of the Bohm potential in the QHD theory requires application of kinetic correction-factor in order for QHD to be fully consistent with that of kinetic theory [40-43]. By the direct use of the Lindhard dielectric function it was confirmed that there is a potential minimum around the shielded charge for specific metallic density regime beyond which Friedel oscillations dominate the Lennard-Jones-like potential [40]. It should be noted that the expansion of the fully quantum kinetic dielectric theory of Lindhard [44] or equivalently the Wigner-Poisson system [40,45] in the zero temperature limit gives different results in two limiting cases. An asymptotic expansion for large phase velocities (small wavenumbers at a finite frequency) gives quantum corrected electron oscillations (cf. Eq. 3.1 in Ref. [45]) consistent with Bohmian theory, while a Taylor expansion for small phase velocities (e.g. zero frequency at a finite wavenumber) yields the shielding by inertialess electrons (cf. Eq. 4.2 in Ref. [45]). This is analogous with classical plasma theory where the asymptotic expansion of the plasma dielectric function gives the Bohm-Gross dispersion relation for electron oscillations, while the low-frequency Taylor expansion gives the linearized Boltzmann response. The theories of Stanton and Murillo [41] and Haas and Mahmood [46] are in the linear limit consistent with such a low-frequency expansion of the quantum kinetic dielectric function for finite electron temperatures.

The aim of this paper is to present a theory of charge shielding for quantum plasmas with arbitrary degeneracy. The model is based on a gradient-corrected Thomas-Fermi model [41] and the independently derived kinetic-modified QHD theory [46], which lead to the same result in the linear regime, and include fully consistent temperature-dependent corrections to the statistical pressure and Bohm potential terms. The paper is organized in the following fashion. The kinetic-modified QHD model is introduced in Sec. II. The LQHD approach with low-frequency expansion approximation is used in Sec. III to derive the static dielectric function for arbitrary degenerate quantum plasma, where the screening potential of a test charge is presented in a more general setting. Applications of the theory to the Thomson scattering cross-section and static structure factor are presented in Sec. VI. Finally, our findings are summarized in Sec. V. 


\section{QUANTUM HYDRODYNAMICS MODEL BEYOND ZERO-TEMPERATURE}

We consider a test charge immersed in a collisionless, dense electron fluid with arbitrary temperature in a homogenous neutralizing static ion background. The number density of the plasma is high enough so that the wave functions of the electrons overlap, which changes the equilibrium electron distribution function to a Fermi-Dirac distribution. Furthermore, we investigate phenomena on atomic scales, where the Bohm potential due to electron tunneling plays an essential role in the electron fluid dynamics. Our treatment of the electron dynamics is based on the finite temperature quantum kinetic theory developed in Ref. [46] for low-frequency excitations of ion-acoustic waves, whose phase speed is much lower than the typical (thermal or quantum statistical) electron speed. The appropriate generalized hydrodynamic equations for the electron number density $n$ and fluid velocity $\mathbf{u}$, coupled with Poisson's equation for the electrostatic potential $\phi$, are given by $[46]$

$$
\begin{aligned}
& \frac{\partial n}{\partial t}+\nabla \cdot(n \mathbf{u})=0, \\
& m_{e} n\left[\frac{\partial \mathbf{u}}{\partial t}+(\mathbf{u} \cdot \nabla) \mathbf{u}\right]=e n \nabla \phi-\nabla P(n)+\frac{\gamma n \hbar^{2}}{6 m_{e}} \nabla[\Delta \sqrt{n} / \sqrt{n}], \\
& \Delta \phi=4 \pi e\left[n-n_{0}-(Q / e) \delta(\mathbf{r})\right],
\end{aligned}
$$

where $e$ is the magnitude of the electron charge, $m_{e}$ is the electron mass, $\hbar$ is Planck's constant divided by $2 \pi, n_{0}$ is a neutralizing ion background density, $P(n)$ is the statistical pressure and $Q=Z e$ is a test charge where $Z$ is the charge state. The fluid theory is developed by expansion of kinetic theory in the long wavelength limit, keeping only dispersive effects due to the statistical pressure $P$ and the so-called Bohm potential, the last term in the right-hand side of Eq. (1b). The pre-factor to the Bohm potential derived from kinetic theory is [46]

$$
\gamma=\frac{\operatorname{Li}_{3 / 2}\left[-\exp \left(\beta \mu_{0}\right)\right] \mathrm{Li}_{-1 / 2}\left[-\exp \left(\beta \mu_{0}\right)\right]}{\operatorname{Li}_{1 / 2}\left[-\exp \left(\beta \mu_{0}\right)\right]^{2}}
$$

where $\beta=1 /\left(k_{B} T\right), T$ is the electron temperature, $k_{B}$ is Boltzmann's constant, $\mu_{0}$ is the equilibrium chemical potential, and $\operatorname{Li}_{s}(z)$ is the polylogarithm with index $s$ and argument $z$. The generalized finite temperature equation of state is defined through moments of 
Fermi-Dirac distribution of the electrons as

$$
\begin{aligned}
& n(\mu, T)=\frac{2^{1 / 2} m_{e}^{3 / 2}}{\pi^{2} \hbar^{3}} \int_{0}^{\infty} \frac{\varepsilon^{1 / 2} d \varepsilon}{e^{\beta(\varepsilon-\mu)}+1}=-\frac{m_{e}^{3 / 2} \operatorname{Li}_{3 / 2}[-\exp (\beta \mu)]}{\sqrt{2} \pi^{3 / 2} \hbar^{3} \beta^{3 / 2}}, \\
& P(\mu, T)=\frac{2^{3 / 2} m_{e}^{3 / 2}}{3 \pi^{2} \hbar^{3}} \int_{0}^{\infty} \frac{\varepsilon^{3 / 2} d \varepsilon}{e^{\beta(\varepsilon-\mu)}+1}=-\frac{m_{e}^{3 / 2} \operatorname{Li}_{5 / 2}[-\exp (\beta \mu)]}{\sqrt{2} \pi^{3 / 2} \hbar^{3} \beta^{5 / 2}}
\end{aligned}
$$

where, $\varepsilon$ is the non-relativistic kinetic energy of the electrons, and use were made of the Fermi-Dirac integrals

$$
I_{s}(y)=\int_{0}^{\infty} \frac{x^{s}}{\exp (x-y)+1} \mathrm{~d} x=-\Gamma(s+1) \operatorname{Li}_{s+1}\left(-\mathrm{e}^{y}\right), \quad s>-1,
$$

where $\Gamma$ is the Gamma function. In the following calculations, the electrons are assumed to be isothermal, i.e. the temperature $T$ is assumed fixed throughout the plasma. Then it can be shown using Eqs. (3) that $(1 / n) \nabla P=\nabla \mu[47]$. In the zero temperature limit, we have $\mu=E_{F e}$, where $E_{F e}=\hbar^{2}\left(3 \pi^{2} n\right)^{2 / 3} /\left(2 m_{e}\right)$ is the Fermi energy. The relation between the equilibrium density and chemical potential is obtained by using $n=n_{0}$ and $\mu=\mu_{0}$ in Eq. (3a). Conventionally, other parameters like the Wigner-Seitz radius $a=\left(3 / 4 \pi n_{0}\right)^{1 / 3}$, the degeneracy parameter $\theta=T / T_{F e}\left(T_{F e}=E_{F e} / k_{B}\right.$ being the Fermi temperature) the coupling constant in the classical limit $\Gamma_{e}=e^{2} /\left(a k_{B} T\right)$ and degenerate limit (the Brueckner parameter) $r_{s}=e^{2} /\left(a E_{F e}\right) \sim m_{e} e^{2} a / \hbar^{2}$ are used to describe the electron gas. The electrons are weakly coupled when $\Gamma \ll$ 1 or $r_{s} \ll 1$ and strongly coupled in the opposite limit. Also we have $r_{s}=$ $\left(n_{0} / 1.6 \times 10^{24}\right)^{-1 / 3}$ and $\theta=2(4 / 9 \pi)^{2 / 3} Z^{5 / 3} r_{s} / \Gamma_{i}$. For $r_{s} \gg 10^{-2}$ the electron fluid can be regarded as nonrelativistic degenerate. The conduction electrons in metals with $r_{s} \simeq 2-6$ are then regarded as strongly coupled plasmas which satisfy the complete degeneracy $\theta \ll 1$ condition [11]. On the other hand, semiconductors with $\theta \geq 1$ and $n_{0}<10^{18} \mathbf{c m}^{-3}\left(r_{s}>25\right)$ are only partially degenerate plasmas and should be treated in the framework of finite temperature Fermi-Dirac statistical theory. The theory of screening presented here is valid for the whole range of nonrelativistic plasma density and temperatures.

Figure 1 shows the variation of the electron number density as a function of the equilibrium chemical potential for different electron temperatures. It is seen that for all temperature values the number density increases with increasing chemical potential. Since the chemical potential of a Fermi gas defines the energy needed to add an extra fermion to the gas, the 


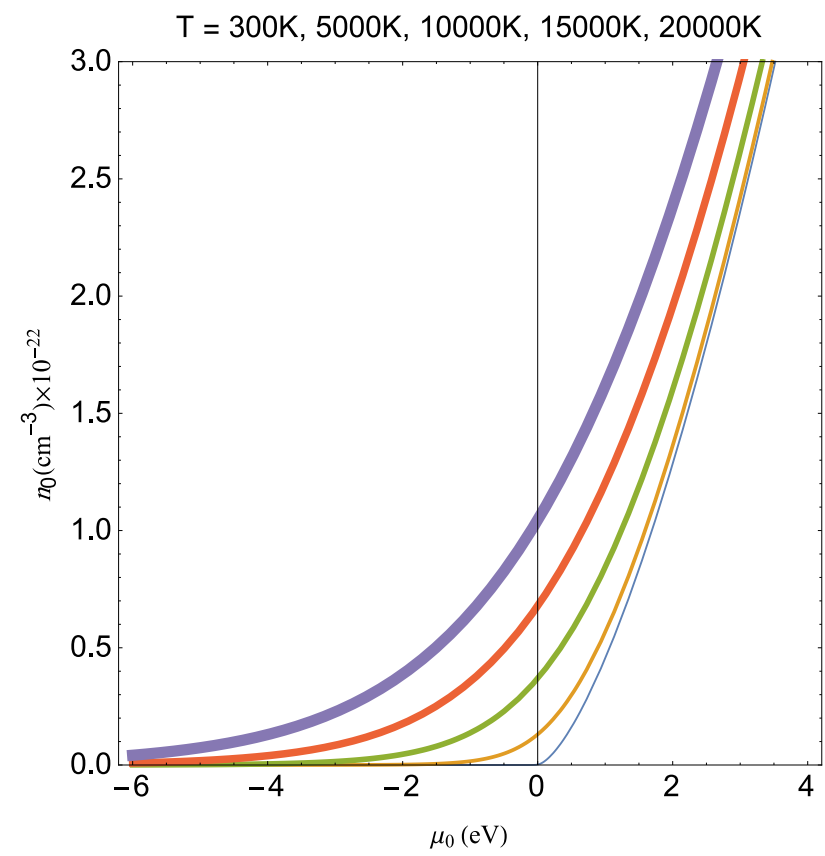

(a)

FIG. 1: The equilibrium electron number density $n_{0}$ as a function of the equilibrium chemical potential $\mu_{0}$ for different values of the electron temperature $T$. Increasing line thickness indicates increasing values of the varied parameter given above the panel.

negative chemical potential refers to the classical limit where there are vacant levels below the Fermi level to absorb extra fermions. However, as the temperature is lowered much below the Fermi temperature, the chemical potential becomes positive (see e.g. the plot for $T=300 \mathrm{~K}$ in Fig. 1). This occurs due to the fact that all the fermions sink to the minimum available fermion energy levels filling all the possible vacant states, and hence, $\mu_{0}$ becomes positive.

In the following section, we will use Eq. (1) in the static limit $\partial / \partial t=0$ and $\mathbf{u}=0$, which is consistent with the $\omega=0$ expansion of kinetic theory, in order to investigate the charge shielding.

\section{DIELECTRIC FUNCTION AND STATIC SCREENING}

In order to derive the linear response of the plasma to the test charge $Q$ in Eq. (1c), we express the unknowns as $n=n_{0}+n_{1}, u=u_{1}, \phi=\phi_{1}$ and $\mu=\mu_{0}+\mu_{1}$, where quantities with subscript ' 0 ' are equilibrium quantities and the ones with subscript ' 1 ' are small linear 
perturbations. The linear quantities are then assumed proportional to plane wave solutions of the form $\exp (i \mathbf{k} \cdot \mathbf{r}-i \omega t)$, where $\mathbf{k}$ is the wave vector and $\omega$ is the frequency. We use the identity $(1 / n) \nabla P=\nabla \mu$ together with the relation Eq. (3) to Fourier analyze the system (1). This gives rise to a dielectric function $D_{\gamma}(\mathbf{k}, \omega)$, which in the static $(\omega=0)$ limit takes the form

$$
D_{\gamma}(\mathbf{k}, 0)=1+\frac{\omega_{p e}^{2}}{v_{T}^{2} k^{2}+\gamma \hbar^{2} k^{4} / 12 m_{e}^{2}} .
$$

where $\omega_{p e}=\sqrt{4 \pi n_{0} e^{2} / m_{e}}$ is the electron plasma frequency, and

$$
v_{T}^{2}=\frac{k_{B} T}{m_{e}} \frac{\operatorname{Li}_{3 / 2}\left[-\exp \left(\beta \mu_{0}\right)\right]}{\mathrm{Li}_{1 / 2}\left[-\exp \left(\beta \mu_{0}\right)\right]}, \quad n_{0}=-\frac{m_{e}^{3 / 2} \operatorname{Li}_{3 / 2}\left[-\exp \left(\beta \mu_{0}\right)\right]}{\sqrt{2} \pi^{3 / 2} \hbar^{3} \beta^{3 / 2}},
$$

where $v_{T}$ is a generalized thermal velocity that takes into account the arbitrary electron degeneracy. It is noted that the definition of thermal speed in Eq. (6) is slightly different from the conventional. The traditional definition of the thermal speed uses the average kinetic energy $(K)$ of the electron gas defined as [46]

$$
v_{T}^{2}=\frac{2\langle K\rangle}{3 m_{e}}=\frac{k_{B} T}{m_{e}} \frac{\mathrm{Li}_{5 / 2}\left[-\exp \left(\beta \mu_{0}\right)\right]}{\mathrm{Li}_{3 / 2}\left[-\exp \left(\beta \mu_{0}\right)\right]},
$$

where in the fully degeneracy limit $\left(\mu_{0} \rightarrow \infty\right)$ one obtains $v_{T}^{2}=v_{F e}^{2} / 3$ in our definition and $v_{T}^{2}=v_{F e}^{2} / 5$ in the conventional one. Using that $\lim _{x \rightarrow \infty} \operatorname{Li}_{s}\left(-e^{x}\right)=$ $-x^{s} / \Gamma(s+1)$, we have that $\lim _{x \rightarrow \infty}\left[\operatorname{Li}_{3 / 2}\left(-e^{x}\right) / x \operatorname{Li}_{1 / 2}\left(-e^{x}\right)\right]=2 / 3$ and $\lim _{T \rightarrow 0} \mu_{0}=E_{F e}$, in the zero temperature degenerate limit $\left(\beta \mu_{0} \gg 1\right)$, we recover $v_{T}=v_{F e} / \sqrt{3}$, with $v_{F e}=\sqrt{2 E_{F e} / m_{e}}$ being the Fermi velocity, and $\gamma=1 / 3$ in the dielectric function given by Eq. (5) $[7,32,40,45]$. In the limit $\beta \mu_{0} \ll-1$ (the classical limit) we have that $\operatorname{Li}\left[-\exp \left(\beta \mu_{0}\right)\right] \approx-\exp \left(\beta \mu_{0}\right)$ so that $\gamma=1$ and $v_{T}=\sqrt{k_{B} T / m_{e}}$. In the limit of high electron temperatures, the $k^{4}$-term becomes negligible compared to that of the $k^{2}$-term, and the dielectric function given by Eq. (5) reduces to the classical one. It is possible to generalize the above dielectric function to include the electron exchange-correlation effects by the transformation $v_{T}^{2} \rightarrow v_{T}^{2}+v_{x c}^{2}$, where $v_{x c}=-\left(0.328 e^{2} n_{0}^{1 / 3} / m_{e}\right)^{1 / 2}\left[1+0.62 /\left(1+18.36 r_{B} n_{0}^{1 / 3}\right)\right]^{1 / 2}[\mathbf{2 4}, \mathbf{2 5}]$ and $r_{B}=\hbar^{2} /\left(m_{e} e^{2}\right) \approx$ $5.29 \times 10^{-9} \mathrm{~cm}$ is the Bohr radius. The exchange-correlation potential has been used to study the optical properties of $\mathrm{Ga}_{1-x} \mathrm{Al}_{x} \mathrm{As}$ semiconductor system in the local density approximation (LDA) where it is assumed to be dependent only on the local electron number density [25]. However, for simplicity we neglect the 
exchange-correlation effects below. On the other hand, in order to evaluate the static charge shielding in the low-frequency limit, we use the gamma-corrected dielectric function with the correction applying to the quantum diffraction term [46], to find

$$
\phi(r)=\frac{Z e}{2 \pi^{2}} \int \frac{\exp (i k r)}{k^{2} D_{\gamma}(k, 0)} d^{3} k .
$$

The evaluation of the integrals leads to the following general form [32]

$$
\phi(r)=\frac{Q}{2 r}\left[(1+b) e^{-k_{+} r}+(1-b) e^{-k_{-} r}\right],
$$

with $k_{ \pm}^{2}=k_{0}^{2}(1 \mp \sqrt{1-4 \alpha}) /(2 \alpha)$, where

$$
k_{0}=\frac{\omega_{p e}}{v_{T}}=\left(\frac{8}{\pi}\right)^{1 / 4} \sqrt{-\frac{m_{e}^{3 / 2} e^{2} \operatorname{Li}_{1 / 2}\left[-\exp \left(\beta \mu_{0}\right)\right]}{\beta^{1 / 2} \hbar^{3}}} .
$$

and the quantum diffraction parameter

$$
\alpha=\frac{\gamma \hbar^{2} \omega_{p e}^{2}}{12 m_{e}^{2} v_{T}^{4}}=-\frac{\alpha_{F} c \sqrt{\beta m_{e}}}{3 \sqrt{2 \pi}} \mathrm{Li}_{-1 / 2}\left[-\exp \left(\beta \mu_{0}\right)\right],
$$

where $\alpha_{F}=e^{2} /(\hbar c) \approx 1 / 137$ is the fine-structure constant and $c$ is the speed of light in vacuum. The static ionic interaction potential given in Eq. (9) can be put into three categories, where $\alpha>1 / 4$ admits an attractive interaction, $\alpha<1 / 4$ leads to a purely repulsive interaction, and the limiting case $\alpha=1 / 4$, as [32]

$$
\phi(r)=\left\{\begin{array}{cc}
\phi_{r e p}(r)=(Z e / r) \exp (-A r)[\cosh (B r)+b \sinh (B r)], \quad \alpha<\frac{1}{4}, \quad 1<b<\infty, \\
\phi_{a t t}(r)=(Z e / r) \exp \left(-A^{\prime} r\right)\left[\cos \left(B^{\prime} r\right)+b^{\prime} \sin \left(B^{\prime} r\right)\right], \quad \alpha>\frac{1}{4}, \quad 0<b^{\prime}<\infty, \\
\phi_{1 / 4}(\mathrm{r})=(Z e / r) \exp \left(-\sqrt{2} k_{0} r\right)\left[1+(\sqrt{2} / 2) k_{0} r\right], \quad \alpha=\frac{1}{4}, \quad b \rightarrow \infty,
\end{array}\right.
$$

where the constants are defined as

$$
A=k_{0} \frac{\sqrt{\sqrt{4 \alpha}+1}}{\sqrt{4 \alpha}}, \quad B=k_{0} \frac{\sqrt{1-\sqrt{4 \alpha}}}{\sqrt{4 \alpha}}, \quad b=\frac{1}{\sqrt{1-4 \alpha}},
$$

and

$$
A^{\prime}=k_{0} \frac{\sqrt{\sqrt{4 \alpha}+1}}{\sqrt{4 \alpha}}, \quad B^{\prime}=k_{0} \frac{\sqrt{\sqrt{4 \alpha}-1}}{\sqrt{4 \alpha}}, \quad b^{\prime}=\frac{1}{\sqrt{4 \alpha-1}} .
$$

In the limit $\alpha=0$, the potential turns to the Debye-Hückel potential with $\phi_{D H}=$ $(Z e / r) \exp \left(-k_{0} r\right)$, which becomes the Thomas-Fermi potential for fully degenerate electrons in the limit $\beta \mu_{0} \gg 1$.

Figure 2 shows the variation of the quantum diffraction parameter $\alpha$ as a function of the equilibrium chemical potential and temperature when one of the parameters is fixed and the 


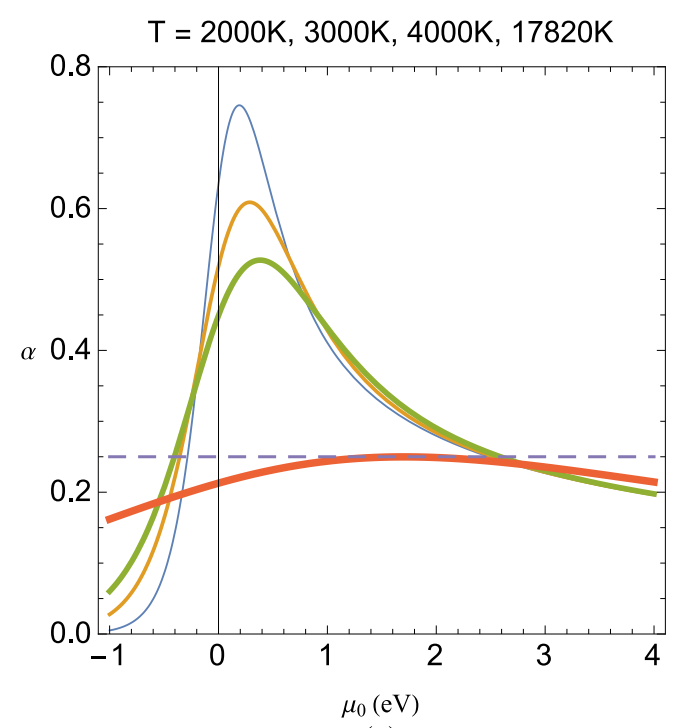

(a)

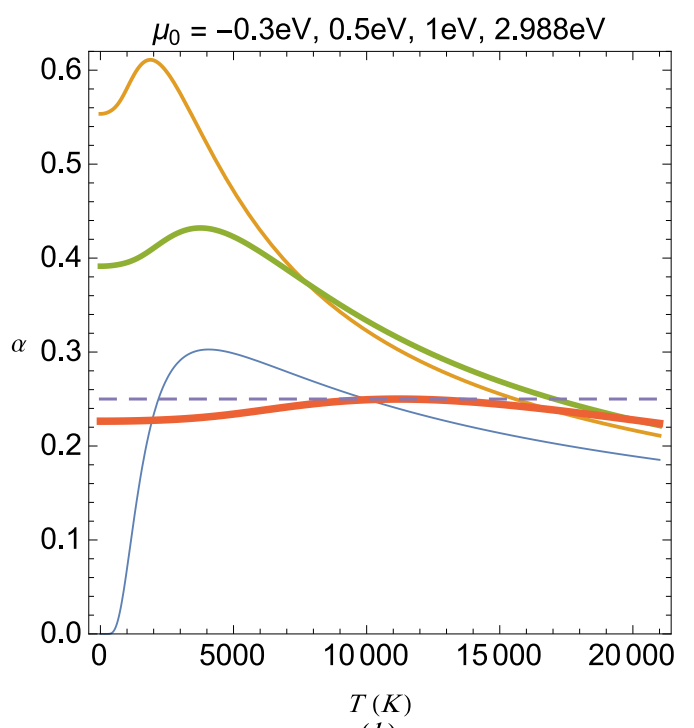

(b)

FIG. 2: (a) The interaction potential parameter $\alpha$ as a function of the equilibrium chemical potential $\mu_{0}$ for different values of electron temperature $T$, and (b) as a function of $T$ for different values of $\mu_{0}$. Increasing line thickness indicates increasing values of the varied parameter given above each panel. The horizontal lines represent the critical value $\alpha=1 / 4$, above which there exists an attractive potential.

other is varied. It is observed from Fig. 2(a) that for every given value of the temperature, in the classical limit $\mu_{0} \rightarrow-\infty, \alpha$ tend to zero and the screening potential tends to the Debye-Hückel potential in classical plasmas. Moreover, there exists a maximum value of the screening potential parameter for a given temperature the position/magnitude of which slightly increases/decreases with increase in the plasma temperature. The horizontal dashed line in Fig. 2(a) separates the two domains of attractive $(\alpha>1 / 4)$ and repulsive $(\alpha<1 / 4)$ screening potentials. We note that $\alpha$ has a maximum value at higher $\mu_{0}$ for higher values of $T$, up to a maximum critical temperature of $T_{c} \simeq 17820 \mathrm{~K}$ with the maximum value of $\alpha$ at $\mu_{0} \simeq 1.6 \mathrm{eV}$. For $T>T_{c}$, the screening becomes purely repulsive. Figure $2(\mathrm{~b})$, on the other hand, shows that the attractive screening potential exists for $\mu_{0}<2.988 \mathrm{eV}$ the maximum value of which coincides with $T \simeq 11420 \mathrm{~K}$. Figure 3 shows that the attractive potential, for which $\alpha>1 / 4$, exists in a bounded region in the $T$ - $\mu_{0}$ plane. The attractive screening region exists for positive values of the chemical potential, and also extends to slightly negative values corresponding to less dense plasmas. In the zero temperature limit the value of the quantum 


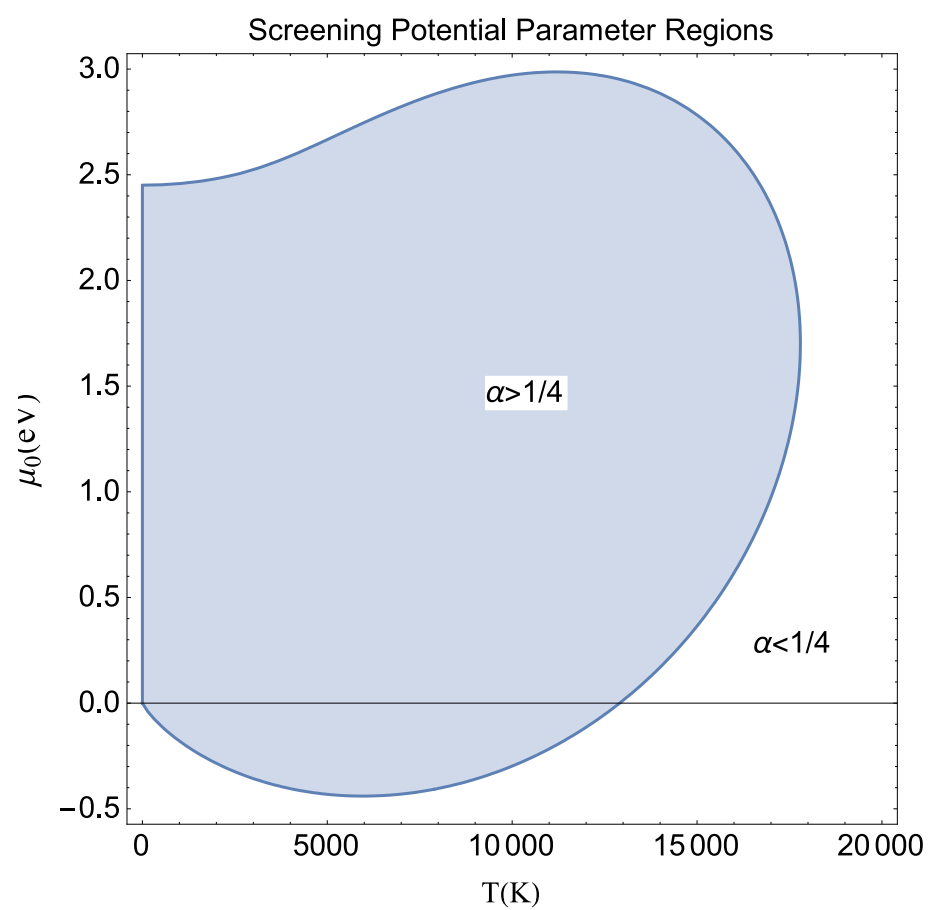

FIG. 3: The existence diagram for the attractive inter-ionic potential (i.e. $\alpha>1 / 4$ ) in the $T$ - $\mu_{0}$ plane. The equilibrium chemical potential and electron temperature is given in units of electron volts and Kelvin, respectively.

diffraction parameter reduces to $\alpha \approx \hbar^{2} \omega_{p e}^{2} /\left(16 E_{F e}^{2}\right) \simeq 6.48 \times 10^{6} n_{0}^{-1 / 3}$, which for $\alpha=1 / 4$ gives the limiting density of $n_{0}=1.74 \times 10^{22} \mathrm{~cm}^{-3}$, corresponding to an electron Fermi energy of $2.45 \mathrm{eV}$. As a comparison, typical metallic densities such as in silver $\left(n_{0}=5.8 \times 10^{22} \mathrm{~cm}^{-3}\right.$, $E_{F}=5.5 \mathrm{eV}$ ) would give repulsive shielding potentials.

Let us compare our results with the finite temperature quantum plasma screening theory based on the gradient-corrected orbital-free DFT [41, 43]. Using atomic units in which $e=m_{e}=\hbar=1$, the screening potential for $\nu<1$ is given as [41]

$$
\phi_{S M}(r)=\frac{Z^{*}}{2 r}\left[\left(1+\alpha_{S M}\right) e^{-r / \lambda_{-}}+\left(1-\alpha_{S M}\right) e^{-r / \lambda_{+}}\right],
$$

which is of similar form as Eq. (9) but with different parameters

$$
\alpha_{S M}=\frac{1}{\sqrt{1-\nu}}, \quad \lambda_{ \pm}^{2}=\frac{\nu \lambda_{T F}^{2}}{2 \pm 2 \sqrt{1-\nu}}, \quad \lambda_{T F}^{2}=\frac{\pi \sqrt{2 \beta}}{4 I_{-1 / 2}\left(\eta_{0}\right)},
$$

where their quantum diffraction parameter $\nu$ is given by

$$
\nu=\frac{3 \lambda \sqrt{8 \beta}}{\pi} \frac{d I_{-1 / 2}\left(\eta_{0}\right)}{d \eta_{0}}=-\frac{4 \sqrt{\beta}}{3 \sqrt{2 \pi}} \mathrm{Li}_{-1 / 2}\left[-\exp \left(\eta_{0}\right)\right], \quad \eta_{0}=\beta \mu_{0},
$$




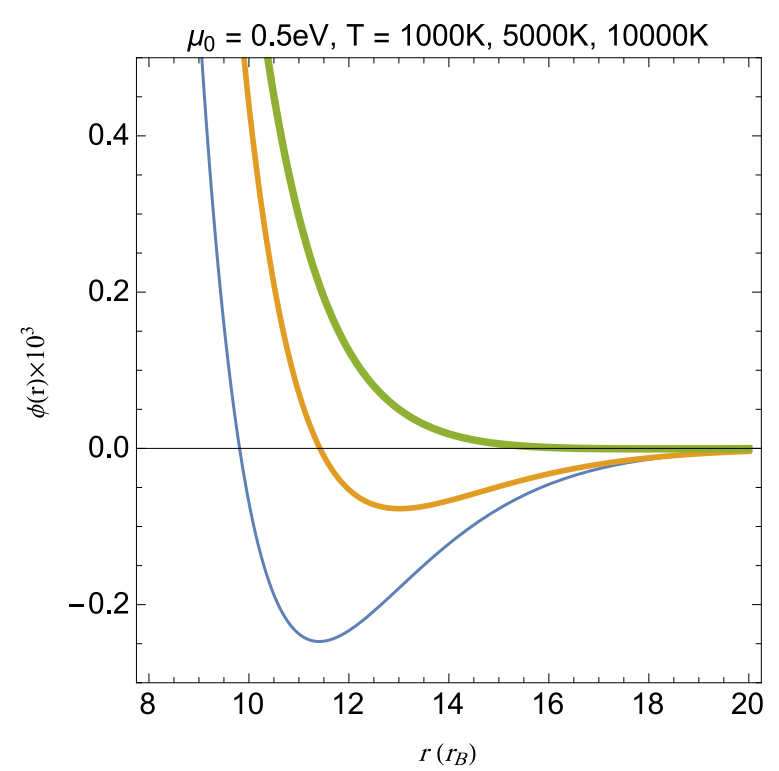

(a)

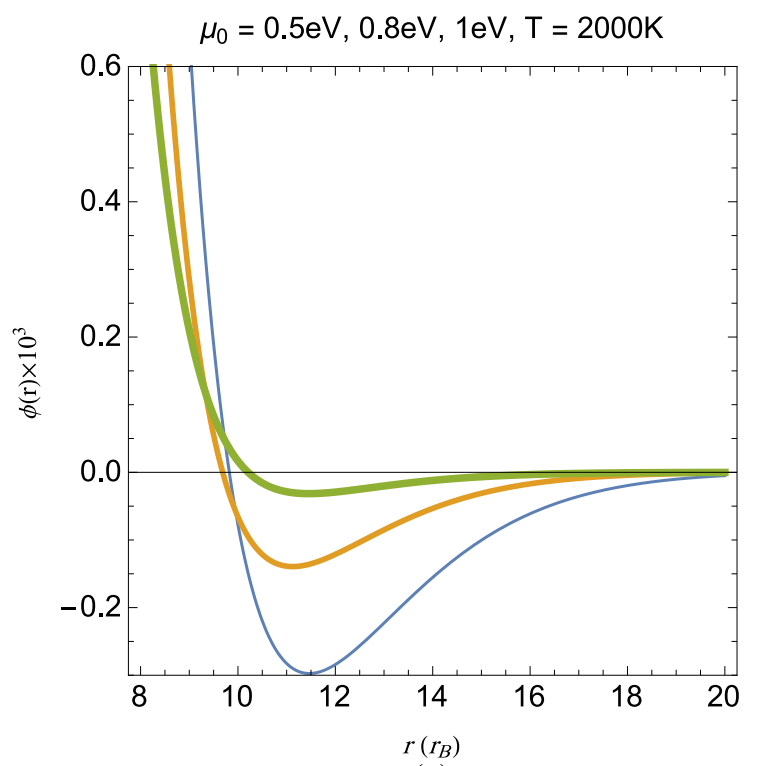

(c)

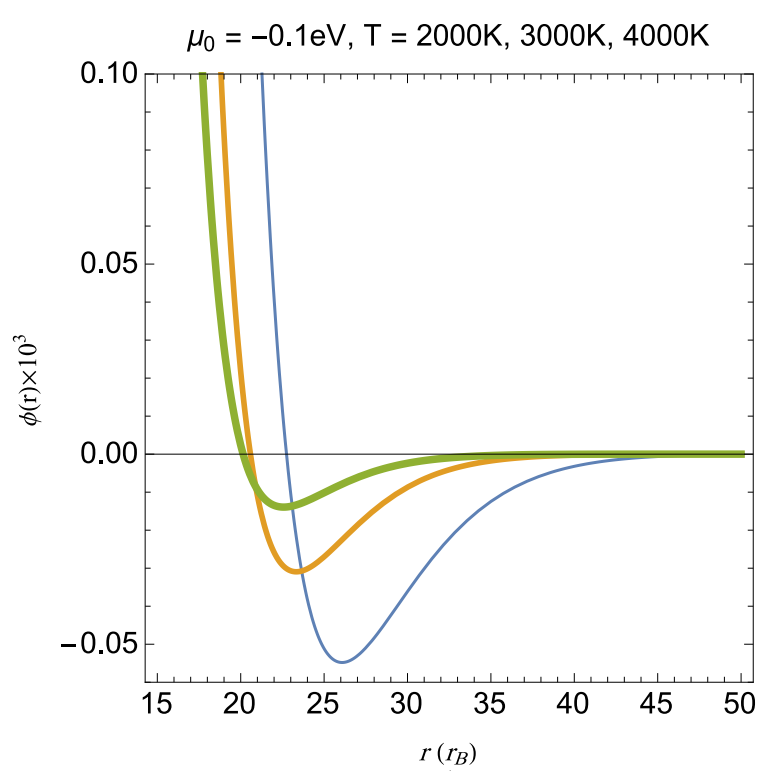

(b)

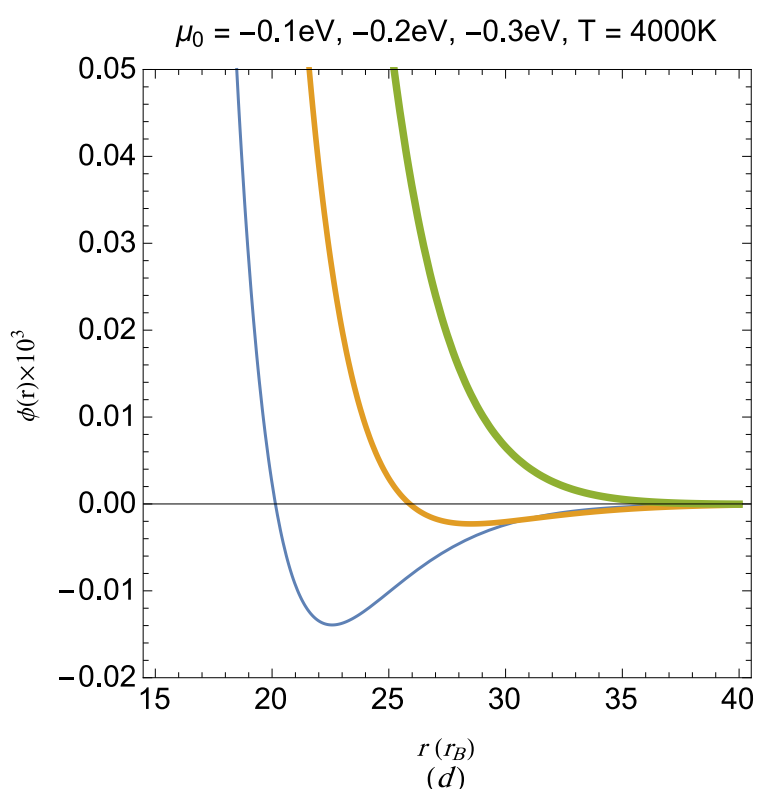

FIG. 4: (a) The spatial profiles of the static screening potential $\phi$ for $\mu_{0}=0.5 \mathrm{eV}$ and different values $T$, (b) for $\mu_{0}=-0.1 \mathrm{eV}$ and different values of $T$, (c) for $T=2000 \mathrm{~K}$ and different positive values of $\mu_{0}$, and (d) for $T=4000 \mathrm{~K}$ and different negative values of $\mu_{0}$. Increasing line thickness indicates increasing magnitudes of the varied parameter given above each panel. The potential is in units of $e / 2 r_{B}$ and is multiplied by a factor $10^{3}$, and the horizontal axis is in unit of the Bohr radius $r_{B}$. Electron number densities for the respective parameter values above the panels are (a) $n_{0} \simeq\{1.7,3,5.7\} \times 10^{21} \mathbf{c m}^{-3}$, (b) $n_{0} \simeq\{2,4.4,7.4\} \times 10^{20} \mathbf{c m}^{-3}$, (c) $n_{0} \simeq\{1.8,3.4,4.7\} \times 10^{21} \mathbf{c m}^{-3}$, and $\left(\right.$ d) $n_{0} \simeq\{7.4,5.8,4.5\} \times 10^{20} \mathbf{c m}^{-3}$. 
with $\lambda=1 / 9$ being the true gradient correction factor in the complete degeneracy limit. Expressing our parameters, $\alpha$ and $k_{0}$ [cf. Eq. (2)] in atomic units gives

$$
\alpha=-\frac{\sqrt{\beta}}{3 \sqrt{2 \pi}} \operatorname{Li}_{-1 / 2}\left[-\exp \left(\eta_{0}\right)\right], \quad k_{0}^{2}=-\frac{\sqrt{8}}{\sqrt{\pi \beta}} \operatorname{Li}_{1 / 2}\left[-\exp \left(\eta_{0}\right)\right] .
$$

It immediately follows that the gradient corrected orbital-free DFT theory [41] and the modified QHD formalism [46] are connected through $\alpha=\nu / 4, k_{0}^{2}=1 / \lambda_{T F}^{2}$ and $k_{ \pm}^{2}=1 / \lambda_{\mp}^{2}$, and hence are completely consistent with each other.

In Fig. 4, the profiles of the charge-screening potential are shown for $Z=1$ and for different values of temperature and chemical potential. In Fig. 4(a) for a positive $\mu_{0}$, the attractive potential valleys are clearly distinguished, the depth of which are observed to decrease with increase of the temperature. It is also observed that equilibrium values of the potential minimum slightly shifts to larger distances $r$ as the temperature is raised, which can be qualitatively interpreted as thermal expansion of the plasma. The profiles in Fig. 4(b), for negative $\mu_{0}$, exhibits very shallow valleys compared to those of Fig. 4(a). These profiles which show the potential for the negative chemical potential region in low-density and high-temperature regime reveal an opposite thermal variation of the screening potentialvalley position compared to that of the positive chemical potentials shown in Fig. 4(a). It is also observed that the increase of the temperature in the negative chemical potential (dilute) regime leads to a decrease in the potential strength. On the other hand, Figs. 4(c) and 4(d) show the dependence of screening profiles with respect to the chemical potential for fixed temperature values for both positive and negative chemical potential regimes. It is seen in Fig. 4(c) that the increase in the positive chemical potential leads to shift of the attractive potential valley depth to smaller distances, opposite to the case of Fig. 4(a). It is also observed that the increase in the chemical potential value (for positive values) decreases the potential valley depth. Figure 4(d) shows that an increase of the negative chemical potential with fixed temperature leads to sharp increase in the effective charge shielding length.

\section{THE THOMSON SCATTERING CROSS-SECTION AND STATIC STRUC- TURE FACTOR}

Some very useful information on ion correlations, phase transition and stopping power in dense quantum plasmas with arbitrary temperature can be obtained using the full dielectric 


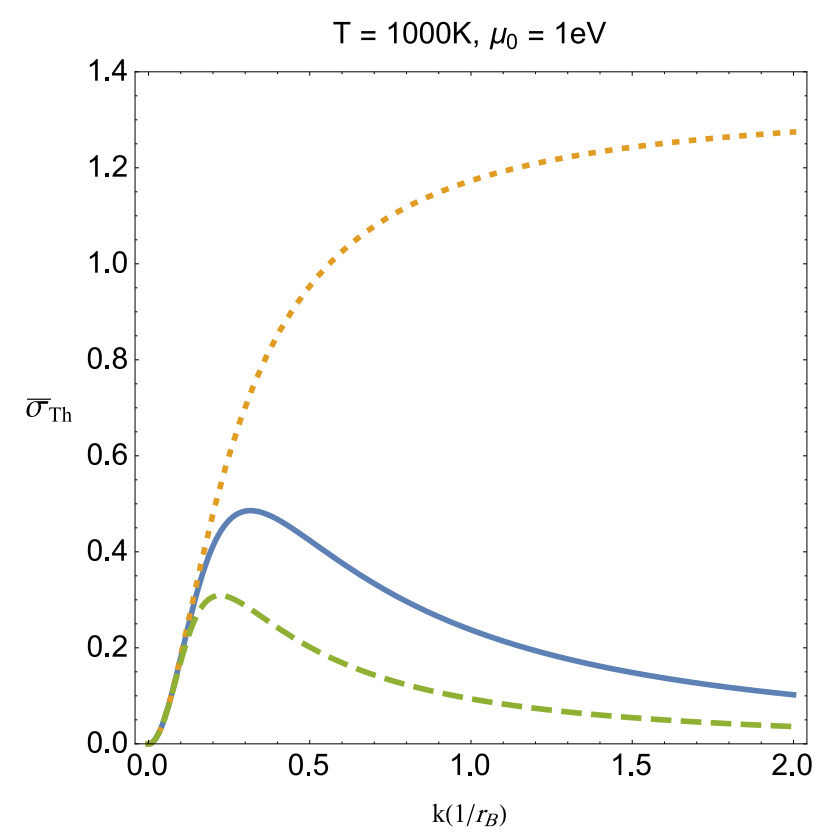

FIG. 5: The normalized Thomson scattering cross section $\bar{\sigma}_{T h}$ as a function of the wavenumber $k$ for $\mu_{0}=1 \mathrm{eV}$ and $T=1000 \mathrm{~K}\left(n_{0} \simeq 4.6 \times 10^{21} \mathbf{c m}^{-3}\right)$, excluding the quantum Bohm potential effect (short-dashed curve), with uncorrected Bohm potential, forcing $\gamma=1$ (long-dashed curve) and with corrected Bohm-potential where $\gamma$ is given by Eq. (2) (solid curve).

function with the detailed calculation of dynamic ion structure factor $S(k, \omega)$ [48]. However, we would like to study an important quantity of dense plasmas known as the Thomson scattering cross-section which can be directly obtained from the static dielectric function in Eq. (5), valid for arbitrary degree of electron degeneracy. The differential Thomson scattering cross-section $d^{2} \sigma_{T h} / d \Omega d \omega$ is directly related to the dynamic structure factor which provides information on density fluctuations in dense plasmas via the fluctuation-dissipation theorem. This quantity defines the scattering of electromagnetic waves off the plasma free electrons in a unit volume $V$ through the solid angle $\Omega$ in the frequency range $d \omega$ and is given by

$$
\frac{d^{2} \sigma_{T h}}{d \Omega d \omega}=V\left(\frac{e^{2}}{m_{e} c^{2}}\right)^{2}\left(\widehat{p}_{i} \cdot \widehat{p}_{f}\right)^{2} S(\mathbf{K}, \omega)
$$

in which $\widehat{p}_{i}$ and $\widehat{p}_{f}$ are the initial and final polarization unit vectors, respectively, and $\mathbf{K}=$ $\mathbf{k}_{\mathbf{f}}-\mathbf{k}_{\mathbf{i}}$ is the momentum transfer vector. The integration over frequency and polarization 


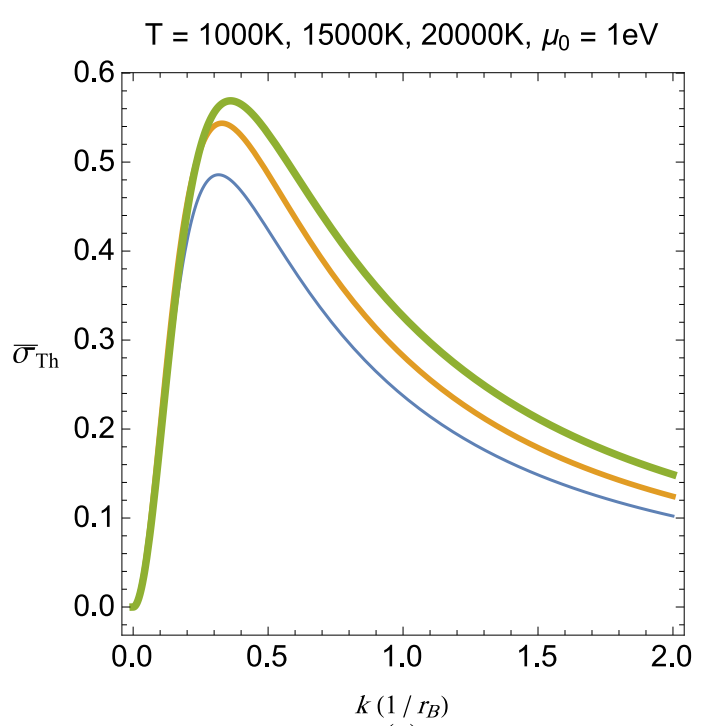

(a)

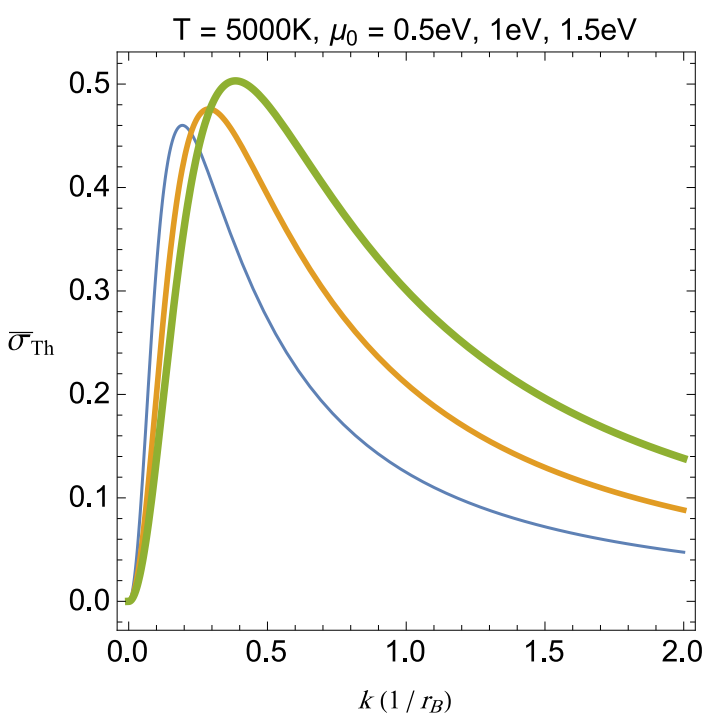

(c)

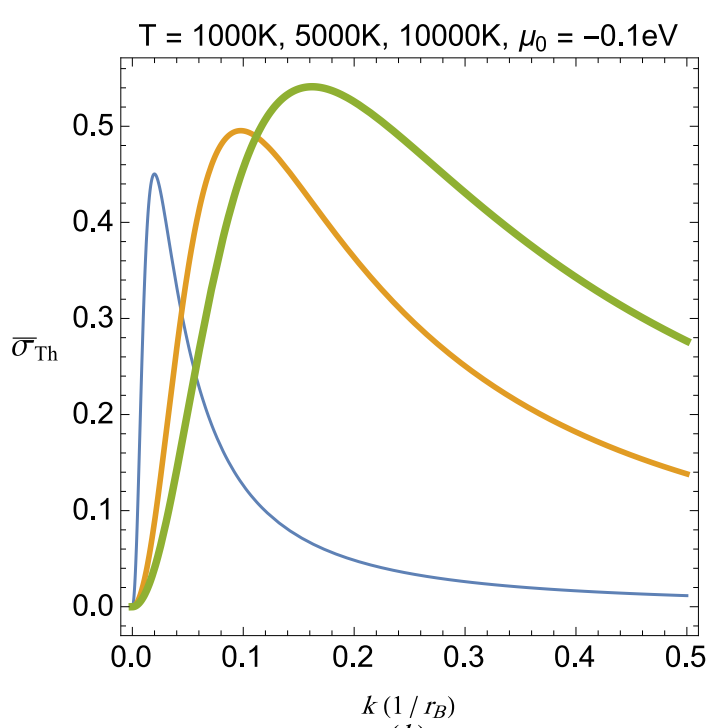

(b)

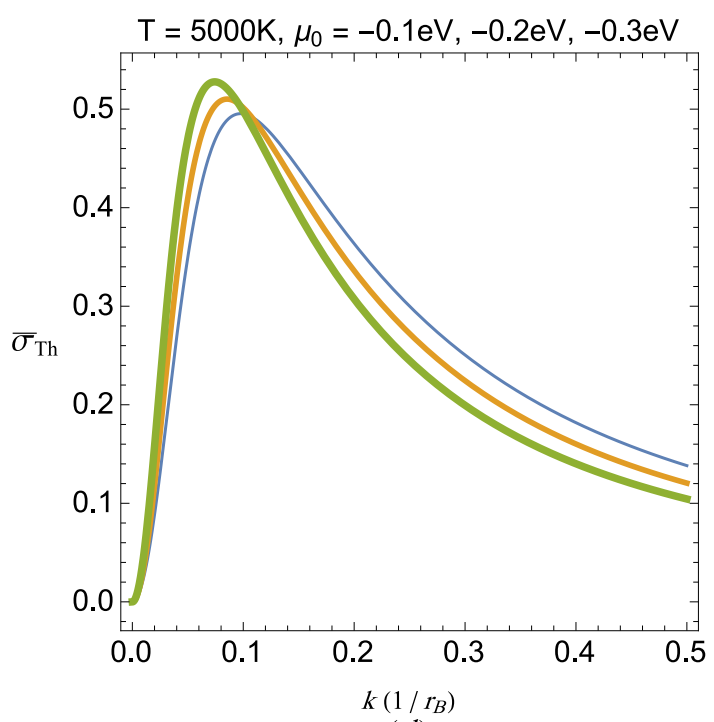

$(d)$

FIG. 6: (a) The normalized Thomson scattering cross section $\bar{\sigma}_{T h}$ as a function of the wavenumber $k$ for $\mu_{0}=1 \mathrm{eV}$ and different values of $T$, (b) for $\mu_{0}=-0.1 \mathrm{eV}$ and different values of $T$, (c) for $T=5000 \mathrm{~K}$ and different values of positive $\mu_{0}$, and (d) for $T=5000 \mathrm{~K}$ and different values of negative $\mu_{0}$. Increasing line thickness indicates increasing magnitudes of the varied parameter given above each panel. The horizontal axis is in units of the inverse Bohr radius, $r_{B}^{-1}$. Electron number densities for the respective parameter values above the panels are (a) $n_{0} \simeq$ $\{4.6,12,16\} \times 10^{21} \mathbf{c m}^{-3}$, (b) $n_{0} \simeq\{4.3,10.8,33.6\} \times 10^{19} \mathbf{c m}^{-3},(\mathbf{c}) n_{0} \simeq\{3,5.7,9.3\} \times 10^{21} \mathbf{c m}^{-3}$, and (d) $n_{0} \simeq\{10.8,8.9,7.3\} \times 10^{20} \mathbf{c m}^{-3}$. 
yields

$$
\frac{d^{2} \sigma_{T h}}{d \Omega}=V\left(\frac{e^{2}}{m_{e} c^{2}}\right)^{2} \frac{1}{2} \sum_{\widehat{p}_{i}, \widehat{p}_{f}}\left(\widehat{p}_{i} \cdot \widehat{p}_{f}\right)^{2} \int_{-\infty}^{\infty} S(\mathbf{K}, \omega) d \omega=N\left(\frac{e^{2}}{m_{e} c^{2}}\right)^{2}\left(1-\frac{1}{2} \sin ^{2} \theta\right) S(\mathbf{K}),
$$

where $N$ is the total number of electrons, $\theta$ is the scattering angle and $S(\mathbf{K})$ is the static structure factor. The momentum transfer vector is then defined as $K^{2}=2 k^{2}(1-\cos \theta)$ assuming elastic scattering. The static structure factor corresponding the dielectric constant Eq. (5) can be written as

$$
S(K, 0)=K^{2}\left[1-\frac{1}{D_{\gamma}(K, 0)}\right]=\frac{K^{2} / k_{0}^{2}}{1+K^{2} / k_{0}^{2}+\alpha K^{4} / k_{0}^{4}} .
$$

The normalized Thomson scattering cross-section is obtained as [49]

$$
\bar{\sigma}_{T h}\left(\mu_{0}, T\right)=\int_{-1}^{1} \frac{K(\xi)^{2} / k_{0}\left(\mu_{0}, T\right)^{2}\left[1-\left(1-\xi^{2}\right) / 2\right] d \xi}{1+K(\xi)^{2} / k_{0}\left(\mu_{0}, T\right)^{2}+\alpha\left(\mu_{0}, T\right) K(\xi)^{4} / k_{0}\left(\mu_{0}, T\right)^{4}}, \quad \xi=\cos \theta .
$$

with $k_{0}\left(\mu_{0}, T\right)$ and $\alpha\left(\mu_{0}, T\right)$ being given by Eq. (10) and (11), respectively. Now, we present a numerical evaluation of the normalized Thomson cross-section obtained from Eq. (22).

Figure 5 shows the effects of the Bohm potential and the modified the Bohm potential on the normalized Thomson scattering cross-section for given values of the chemical potential and the plasma temperature. It is observed that the Thomson cross-section in the absence on Bohm potential (short-dash profile) increases with decrease of the electromagnetic wavelength and extends to very high values where it saturates. This effect however, similar to the ultraviolet catastrophe in Planck radiation problem leads to unphysical situation. Introduction of the electron diffraction effect (long-dash profile) is observed to significantly alters the scattering picture where a maximum values of Thomson cross-section appears at $\lambda_{\max } \simeq 1.075 \mathrm{~nm}$. Also, further change to gamma-corrected modified QHD model (solid profile) leads to an increase of the magnitude of the cross-section and shift of its maximum value to $\lambda_{\max } \simeq 0.71 \mathrm{~nm}$ which well-resides at the soft $\mathrm{x}$-ray spectrum. It is clear that the x-ray Thomson scattering spectrum may be used to evaluate the correctness of this as well as Stanton-Murillo theory for screening in finite temperature quantum plasmas.

On the other hand, Fig. 6 show the variations of Thomson scattering cross-section profiles as a function of both temperature and the chemical potential. Figure 6(a) indicates that an increase of the temperature with fixed positive chemical potential leads to overall increase in scattering cross-section slightly shifting the maximum scattering value to lower 
wavelengths. The temperature effect for negative chemical potential, in Fig. 6(b) shows a completely different behavior in which the increase of the temperature leads to the pronounced broadening of the profile and also shifting of the maximum scattering values to lower wavelengths. Furthermore, the increase of positive chemical potential for fixed temperature is observed in Fig. 6(c) to lead to the overall increase of the scattering cross-section and shifting the maximum scattering wavelength to lower values. Also, the increase of negative chemical potential in Fig. 6(d) with fixed temperature leads to shifting of the maximum scattering to higher wavelengths, in contrast to the case with positive chemical potential.

\section{SUMMARY}

The charge shielding problem has been investigated in the framework of linearized quantum hydrodynamic model [46] consistent with gradient-corrected orbital-free DFT [41], which account for finite temperature and including the kinetic correction for the electron quantum diffraction effect. The correct hydrodynamic limit discussed here can be obtained by a low-frequency, low phase velocity expansion of kinetic theory, in contrast to the high phase velocity small wavenumber limit, which gives rise to high-frequency electron oscillations consistent with Bohmian theory. It is found that the screening potential profile significantly depends on the temperature and chemical potential of plasma. The variation of attractive potential valley in terms of the changes in temperature can qualitatively describe the plasma thermal expansion and ion-ion interactions for a wide range of temperaturechemical-potential regimes extending from completely degenerate to dilute quantum plasmas. The Thomson scattering was also found to be a function of plasma temperature and chemical potential and is fundamentally affected by the Bohm potential and kinetic gammacorrection. It was particularly shown that the characteristics of the charge shielding and Thomson scattering in two distinct regimes of negative and positive chemical potential values are completely different. Current study may be useful in parametric study of diverse physical properties of fermion plasmas with arbitrary degeneracy and the calculated screening 
potentials may be used in molecular-dynamic simulations of quantum plasmas.

[1] P.A. Markovich, C.A. Ringhofer, and C. Schmeister, Semiconductor Equations, (Springer, Berlin, 1990).

[2] H. Haug and S. W. Koch, Quantum Theory of the Optical and Electronic Properties of Semiconductors (World Scientific, Singapore, 2004).

[3] M. Meyyappan, J. Phys. D: Appl. Phys. 44, 174002 (2011).

[4] S. H. Glenzer and R. Redmer, Rev. Mod. Phys. 81, 1625 (2009).

[5] R. H. Fowler, Mon. Not. Roy. Astr. Soc. 87, 114 (1926).

[6] Y. L. Klimontovich and V. P. Silin, Dokl. Akad. Nauk SSSR 82 361(1952); ibid. Zh. Eksp. Teor. Fiz. 23, 151 (1952).

[7] D. Bohm, Phys. Rev. 85, 166 (1952).

[8] D. Bohm and D. Pines, Phys. Rev. 92, 609 (1953).

[9] D. Bohm and D. Pines, in Plasma Physics, Ed. J. E. Drummond (McGraw-Hill, New York, 1961).

[10] D. Pines, J. Nucl. Energy: Part C: Plasma Phys. 2, 5 (1961).

[11] S. Ichimaru, Rev. Mod. Phys. 54, 1017 (1982).

[12] S. Ichimaru, H. Iyetomi and S. Tanaka, Phys. Rep. 149, 91 (1987).

[13] N. W. Ashcroft and N. D. Mermin, Solid State Physics (Saunders College Publishing, Orlando, 1976).

[14] V. E. Fortov, Phys. Usp. 52, 615 (2009).

[15] S. Chandrasekhar, An Introduction to the Study of Stellar Structure (The University of Chicago press, Chicago, 1939).

[16] E. E. Salpeter, Astrophys. J. 134, 669 (1961).

[17] D. Kremp, M. Schlanges, and W.-D. Kraeft, Quantum Statistics of Nonideal Plasmas (Springer Verlag, Berin, 2005).

[18] C. L. Gardner, C. Ringhofer, Phys. Rev. E 53, 157 (1996).

[19] G. Manfredi, Fields Inst. Commun. 46, 263 (2005).

[20] F. Haas, Quantum Plasmas: An Hydrodynamic Approach (Springer, New York, 2011).

[21] Y.-D. Jung, Phys. Lett. A 378, 2176 (2014). doi: 10.1016/j.physleta.2014.06.008 
[22] Y.-D. Jung and W.-P. Hong, Phys. Plasmas 20, 084502 (2013).

[23] B. Eliasson and P. K. Shukla, Phys. Scr. 78, 025503 (2008).

[24] L. Hedin and B. I. Lundqvist, J. Phys. C 4, 2064 (1971).

[25] L. Brey, J. Dempsey, N. F. Johnson, and B. I. Halperin, Phys. Rev. B. 42, 1240 (1990).

[26] N. Crouseilles, P. A. Hervieux, G. Manfredi, Phys. Rev. B 78, 155412 (2008).

[27] G. Brodin and M. Marklund, New J. Phys. 17, 277 (2007).

[28] J. T. Mendonça, Phys. Plasmas, 18, 062101 (2011).

[29] P. K. Shukla and B. Eliasson, Phys. Usp. 51, 53 (2010).

[30] P. K. Shukla and B. Eliasson, Rev. Mod. Phys. 83, 885(2011).

[31] P. K. Shukla and B. Eliasson, Phys. Lett. A 372, 2897 (2008).

[32] P. K. Shukla and B. Eliasson, Phys. Rev. Lett. 108, 165007 (2012); 108, 219902 (E) (2012); ibid. 109, 019901 (E) (2012).

[33] E. Madelung, Z. f. Physik 40, 332 (1926)

[34] S. K. Ghosh and B. M. Deb, Phys. Rep. 92(1), 1 (1982).

[35] M. Bonitz, E. Pehlke, and T. Schoof, Phys. Rev. E 87, 033105 (2013).

[36] P. K. Shukla, B. Eliasson, and M. Akbari-Moghanjoughi, Phys. Rev. E 87, 037101 (2013).

[37] M. Bonitz, E. Pehlke, and T. Schoof, Phys. Rev. E 87, 037102 (2013).

[38] P. K. Shukla, B. Eliasson and M. Akbari-Moghanjoughi, Phys. Scr. 87018202 (2013).

[39] M. Bonitz, E. Pehlke, and T. Schoof, Phys. Scr. 88, 057001 (2013).

[40] M. Akbari-Moghanjoughi, Phys. Plasmas 22, 022103 (2015); ibid. 22, 039904 (E) (2015).

[41] L. G. Stanton and M. S. Murillo, Phys. Rev. E 91, 033104 (2015); ibid. 91, 049901 (E) (2015).

[42] D. Michta, F. Graziani, and M. Bonitz, Contrib. Plasma Phys. 55, 437 (2015).

[43] Zh. Moldabekov, T. Schoof, P. Ludwig, M. Bonitz, and T. Ramazanov, Phys. Plasmas 22, $102104(2015)$.

[44] J. Lindhard, Dan. Mat. Fys. Medd. 28(8) (1954).

[45] B. Eliasson and P. K. Shukla, J. Plasma Phys. 76, 7 (2010).

[46] F. Haas and S. Mahmood, Phys. Rev. E 92, 053112 (2015).

[47] A. E. Dubinov and I. N. Kitaev, Phys. Plasmas 21, 102105 (2014).

[48] P. K. Shukla and M. Akbari-Moghanjoughi, Phys. Rev. E 87, 043106(2013).

[49] G. W. Lee and Y.-D. Jung, Phys. Plasmas. 20, 062108 (2013). 